\title{
Mucin production determines sensitivity to bortezomib and gemcitabine in pancreatic cancer cells
}

\author{
THADDÄUS TILL WISSNIOWSKI ${ }^{*}$, SILKE MEISTER $^{2^{*}}$, ECKHART G. HAHN $^{3}$, \\ JOACHIM R. KALDEN ${ }^{4}$, REINHARD VOLL $^{4 *}$ and MATTHIAS OCKER ${ }^{*}$ \\ ${ }^{1}$ Division of Gastroenterology, University Hospital, Philipps-University Marburg, Marburg; \\ ${ }^{2}$ IZKF Research Group, Nikolaus-Fiebiger-Center for Molecular Medicine, Erlangen; ${ }^{3}$ Department of Medicine 1, \\ University Hospital Erlangen, Erlangen; ${ }^{4}$ Rheumatology and Clinical Immunology, University Hospital Freiburg, \\ Freiburg; ${ }^{5}$ Institute for Surgical Research, Philipps-University Marburg, Marburg, Germany
}

Received September 13, 2011; Accepted November 14, 2011

DOI: $10.3892 /$ ijo.2012.1337

\begin{abstract}
The prognosis of pancreatic cancer remains disappointing due to a high intrinsic resistance against chemotherapeutic agents. Standard gemcitabine therapies have improved overall survival only marginally and recently, inhibition of the proteasome by the boronic acid derivative bortezomib has been introduced as a novel therapeutic strategy for solid and hematological malignancies including pancreatic cancer. The mucus-producing pancreatic cancer cell line Capan-1 was cultured under standard conditions and treated with different concentrations of gemcitabine or bortezomib. Mucus production was suppressed by siRNA-mediated silencing of apomucin genes. Cell proliferation was determined by ${ }^{3} \mathrm{H}$-thymidine incorporation and apoptosis was quantified after propidium iodide staining by flow cytometry. Apoptotic cell death was confirmed by TUNEL staining, determination of mitochondrial transmembrane potential and assessment of caspase 3/7 activity. NFkB-activity was determined by EMSA. The unfolded protein response (UPR) was further investigated by PCR, Western blotting and caspase 12 activity assays. Silencing of MUC4 significantly reduced expression of mucins for up to 5 days after transfection. While native cells showed an increased sensitivity to bortezomib treatment, silenced cells were more sensitive to gemcitabine treatment. Bortezomib induces mitochondrial damage in native cells and also activates the UPR by splicing of Xbp-1 and induction of CHOP, which is significantly reduced by silencing of MUC4. Our data suggest that mucinous pancreatic cancers are more sensitive
\end{abstract}

Correspondence to: Dr T.T. Wissniowski, Division of Gastroenterology, University Hospital of the Phillips-University Marburg, Baldingerstrasse, 35043 Marburg, Germany

E-mail: wissniowski@me.com

*Contributed equally

Key words: unfolded protein response, endoplasmic reticulum stress, apoptosis, chemotherapy resistance towards proteasome inhibition by bortezomib and that alternative pathways of apoptosis are involved in cell death induction, while tumor cells with a low secretory activity show a better response to gemcitabine.

\section{Introduction}

Pancreatic cancer ranks 4th in cancer related deaths in men and women in the US, with an estimated incidence and death rate of approximately 37,680 and 34,390 cases, respectively (1). Overall survival of advanced disease stages remains disappointing due to the failure of currently available chemotherapy regimens (2-4). Standard therapy consists of combinations of gemcitabine, a pyrimidine-analogue, and other cytotoxic or targeted agents but is still limited by a high intrinsic rate of resistance against these therapies (3-5). Recently, inhibition of the ubiquitin-proteasome pathway by the boronic acid dipeptide derivative bortezomib (BTZ) has been introduced as a novel anticancer therapy in hematologic and solid tumors (6). In contrast to other solid tumors, BTZ alone or in combination with gemcitabine could not significantly prolong overall survival but was associated with grade $3 / 4$ toxicities in a phase II trial or in an orthotopic mouse model $(7,8)$. The outcome of these trials was surprising as a clear molecular rationale for using BTZ in pancreatic cancer was established previously by showing that a variety of cell cycle and apoptosis regulators were regulated by proteasomal degradation (6). Among these factors, the transcriptional inhibitor nuclear factor kappa $\mathrm{B}$ (NFkB), which is closely linked to pancreatic cancer pathogenesis and resistance, has been shown to be a major target of proteasome inhibition (9-11). NFאB becomes activated by phosphorylation and ubiquitination of its inhibitor inhibitor of $\mathrm{NF} \kappa \mathrm{B}$ (I $\kappa \mathrm{B}$ ) by the multi-protein kinase complex inhibitor of $\mathrm{I} \kappa \mathrm{B}$ kinase (IKK) which finally leads to proteasomal degradation of I $\kappa \mathrm{B}$, release and nuclear translocation of $\mathrm{NF \kappa B}$ and target gene activation $(12,13)$. This pathway is activated in most pancreatic cancer cell lines or clinical specimens and is also correlated with poor survival and apoptosis resistance due to overexpression of anti-apoptotic genes $(14,15)$. In preclinical models, inhibition of NFKB has thus been shown to sensitize pancreatic cancer cells to death-receptor or chemotherapy 
induced apoptosis (16-18). Recent studies also indicate a role of the unfolded protein response (UPR) and endoplasmic reticulum (ER)-mediated stress pathways in induction of apoptosis by BTZ, probably due to interference with molecular chaperones like HSP70 and $\operatorname{BiP}(19,20)$.

Mucins, like other secreted proteins, are dependent on correct processing by chaperones in the ER (21). We have shown previously that the effect of BTZ in myeloma cells is also dependent on the secretory activity (22). In pancreatic cancer, high expression of mucins has been linked to resistance against chemotherapy, tumor progression and an aggressive phenotype (23-27). We therefore investigated if and to what extent the inhibition of mucin production sensitized pancreatic cancer cells to BTZ treatment and compared these findings with conventional gemcitabine therapy. We found a positive correlation between the expression of mucins and BTZ-induced apoptosis, which was predominantly mediated via the ER stress pathway. In addition, a negative correlation with gemacitabine was also observed.

\section{Materials and methods}

Cell culture. The mucus-producing human ductal pancreatic cancer cell line Capan-1 was obtained from the Deutsche Sammlung für Mikroorganismen und Zellkulturen (DSMZ, Braunschweig, Germany) and cultured under standard conditions in RPMI-1640 medium with $20 \%$ (v/v) fetal bovine serum and $1 \%$ penicillin/streptomycin (all from Biochrom, Berlin, Germany). Gemcitabine (Gemzar, Lilly, Bad Homburg, Germany) and bortezomib (Velcade; Janssen-Cilag, Neuss, Germany) were obtained from the pharmacy of the University Hospital Erlangen, Germany.

siRNA transfection. Capan-1 cells were seeded in 6-well flat bottom plates (Nunc, Langenselbold, Germany) at $10^{6}$ cells $/ \mathrm{ml}$ in serum-free medium. siRNA tranfection was performed using Oligofectamine (Invitrogen, Karlsruhe, Germany) according to the manufacturerer's instructions and as described previously (28). All siRNAs were obtained from Qiagen, Hilden, Germany and were targeted against MUC1 (Hs_MUC1_7), MUC2 (Hs_MUC2_1), MUC3 (Hs_LOC642135_3) or MUC4 (Hs_MUC4_11) or were a non-silencing negative control siRNA (AllStars Negative Control). Mock transfections were included for each experiment.

Protein secretion. Samples of the supernatant were collected at $2 \mathrm{~h}$ intervals from transfected cells which were incubated in serum-free medium for $4 \mathrm{~h}$ and stored at $-20^{\circ} \mathrm{C}$ for further measurements. Protein concentration was determined by BCA assay (Pierce, Perbio Science Deutschland GmbH, Bonn, Germany) and measured using a photometer at $595 \mathrm{~nm}$ (Genios, Tecan Germany GmbH, Crailsheim, Germany). The extinction was normalized to a standard curve calculated with dilution series of bovine serum albumin (Sigma-Aldrich, Munich, Germany).

${ }^{3} \mathrm{H}$-thymidine incorporation assay. Capan-1 cells $\left(10^{5}\right)$ were seeded in 96-well round bottom plates (Nunc) and were treated with indicated concentrations of bortezomib or gemcitabine. ${ }^{3} \mathrm{H}$-thymidine $(10 \mu \mathrm{Ci}$ ) (Amersham Pharmacia Biotech, Freiburg,
Germany) was added after $16 \mathrm{~h}$. Cells were transferred to glass fibre sheets (Cell Harvester, Inotech, Dottikon, Switzerland) after an additional $8 \mathrm{~h}$ (total treatment time is $24 \mathrm{~h}$ ). Thymidine incorporation was determined with a $\beta$-imager plate and a BAS reader 5148 (Fuji, Düsseldorf, Germany). Analysis was performed with AIDA software (Raytest, Berlin, Germany).

Isolation of total RNA, reverse transcription and PCR analysis. Total RNA was isolated using RNApure (PeqLab, Erlangen, Germany) according to the manufacturer's protocol. Isolated RNA was resolved in DEPC water (Sigma, Munich, Germany) and concentration was determined photometrically at $260 \mathrm{~nm}$ in a Genios plate reader (Tecan, Crailsheim, Germany). RNA $(1 \mu \mathrm{g})$ was used for reverse transcription using Superscript II reverse transcriptase (Invitrogen, Karlsruhe, Germany), oligodT-primer (Biomol, Berlin, Germany) and random hexamer primer (Promega, Heidelberg, Germany). cDNA was stored at $-20^{\circ} \mathrm{C}$ until use.

Quantitative real-time PCR was performed on the LightCycler system (Roche Diagnostics, Mannheim, Germany) using the DNA Master Kit SYBR green I (Roche Diagnostics) as described previously (29) and QuantiTect Primer assays (Qiagen) for GAPDH (QT01192646) as an internal standard, Bcl-2 (QT00025011), Bax (QT00031192), CHOP (QT00082278), MUC1 (QT01667239), MUC2 (QT01004675), MUC3 [QT01529976(A); QT01679692(B)] and MUC4 (QT00045479).

Regular PCR was performed for determining Xbp-1 splicing using the MasterMix Kit (Fermentas, St. Leon Rot, Germany) on a DNA Engine (PTC-200) Peltier Thermal Cycler (Bio-Rad, Munich, Germany). PCR conditions were $90^{\circ} \mathrm{C}$ for $10 \mathrm{~min}$ and 35 cycles with $57^{\circ} \mathrm{C}$ for $20 \mathrm{sec}$, $70^{\circ} \mathrm{C}$ for $20 \mathrm{sec}$ and $90^{\circ} \mathrm{C}$ for $30 \mathrm{sec}$. Specific primers for Xbp-1 (forward, CCTTGTGGTTGAGAACCAGG; reverse, TCTCTGTCTCAGAGGGGATC) were obtained from MWG Biotech AG (Ebersberg, Germany). PCR products were stained with ethidium bromide (Sigma) and subjected to gel electrophoresis on a $1.7 \%$ (w/v) agarose/TAE gel (Sigma). Bands were visualised under UV light. For these experiments, Capan-1 cells treated for $6 \mathrm{~h}$ with $5 \mu \mathrm{g} / \mathrm{ml}$ of the glycosylation inhibitor tunicamycin (Sigma) were used as a positive control.

Flow cytometry of cell cycle, cell death and mitochondrial transmembrane potential. Treated cells and supernatants were collected and processed as described by staining with hypotonic propidium iodide solution (28). The mitochondrial transmembrane potential $\Delta \Psi_{\mathrm{m}}$ was determined by staining of $10^{5}$ treated cells with 3,3'-dihexyloxacarbocyanine iodide ( $\mathrm{DiOC}_{6}$, Sigma) for $20 \mathrm{~min}$ at $37^{\circ} \mathrm{C} \mathrm{(30).} \mathrm{All} \mathrm{flow} \mathrm{cytometric} \mathrm{analyses} \mathrm{were}$ performed on an Epics Profile Analyzer (Coulter, Miami, FL, USA).

Caspase activity assays. Cells $\left(5 \times 10^{4}\right)$ were treated with bortezomib or gemcitabine as indicated for $16 \mathrm{~h}$. Activity of caspase 3/7 and 8 was determined with Caspase-Glo assays (Promega, Madison, WI, USA) according to the manufacturer's instructions. Luminescence signals were determined on a Genios plate reader (Tecan). The Caspase-12 Fluorimetric Assay Kit (Biocat, Heidelberg, Germany) was used for fluorimetric quantification 
of caspase 12 activity according to the manufacturer's protocol and as described previously (30). Fluorescence signals were measured on a Genios plate reader (Tecan) with excitation at $400 \mathrm{~nm}$ and emission at $505 \mathrm{~nm}$.

TUNEL assay. Cells $\left(10^{6}\right)$ were seeded in chamber slides (Nunc) and were treated as indicated for $24 \mathrm{~h}$. Supernatant was discharged and slides were air-dried at $4^{\circ} \mathrm{C}$. TUNEL staining was performed using the In situ cell death detection Kit (Roche) according to the manufacturer's instructions. Final staining was done with 3,3'-diaminobenzidine (DAB) substrate (Roche). Slides were analyzed by light microscopy.

Protein isolation and Western blotting. Cells $\left(1.5 \times 10^{6}\right)$ were collected and lysed by adding $2 \mathrm{X}$ sample buffer as described (29). Protein concentration was determined by the BCA Protein Assay Reagent (Thermo Fisher Scientific, Bonn, Germany) and measured at $595 \mathrm{~nm}$ in a Genios plate reader (Tecan). Extinction was normalized to a standard curve calculated with dilution series of bovine serum albumin (Sigma).

Samples were incubated at $70^{\circ} \mathrm{C}$ with $10 \%$ reducing agent and $25 \%$ LDS sample buffer (both from Invitrogen) and subjected to electrophoresis on precast $14 \%$ bis-tris-polyacrylamide NuPAGE gels (Invitrogen) at $125 \mathrm{~mA}$ and $200 \mathrm{~V}$ for $50 \mathrm{~min}$. Blotting was performed with transfer buffer (Invitrogen) containing 10\% (v/v) methanol (Sigma) in a cooled blot chamber at $90 \mathrm{~mA}$ and $90 \mathrm{~V}$ for $60 \mathrm{~min}$ on nictrocellulose membranes (Whatman Biometra, Göttingen, Germany). Membranes were blocked with PBS containing $0.1 \%$ Tween-20 (Sigma) and 3.5\% low fat milk for $12 \mathrm{~h}$ at $4^{\circ} \mathrm{C}$ and incubated with the primary antibodies given in Table I for $1 \mathrm{~h}$ at room temperature. After washing 3x for $10 \mathrm{~min}$ with the blocking buffer, appropriate secondary antibodies (Table I) were incubated for $1 \mathrm{~h}$ at room temperature. Reactive bands were visualised with ECL chemiluminescence reagent and detected using X-ray film (both from Amersham Pharmacia Biotech). Results were densitometrically evaluated by AIDA $^{\mathrm{TM}}$ (Raytest, Berlin, Germany).

Electrophoretic mobility shift analyses (EMSA). Electrophoretic mobility shift assays (EMSA) were done as described previously (31). Briefly, nuclear extracts were prepared from $5 \times 10^{6}$ cells treated as indicated. Nuclear extracts $(10 \mu \mathrm{M})$ were added to the probe mix. Probes were end labeled with ${ }^{32} \mathrm{P}-\mathrm{dGTP}$ (Hartmann Analytik, Braunschweig, Germany). The reaction mixtures were incubated at room temperature for $15 \mathrm{~min}$ and run on a $4 \%$ non-denaturing polyacrylamide gel. For specificity control, nuclear extracts were incubated for $15 \mathrm{~min}$ at room temperature with unlabeled double-stranded oligonucleotides or a respective mutant variant at 100 -fold molar excess. Gels were dried on Whatman paper and exposed to X-ray film for $48 \mathrm{~h}$ at $-70^{\circ} \mathrm{C}$. Alternatively, gels were analyzed using phosphoimaging instrumentation (Fujifilm FLA-3000, Agilent Technologies, Palo Alto, CA).

Statistical analysis. Statistical evaluation was done with Microsoft Excel 2003 ${ }^{\mathrm{TM}}$ (Microsoft Corp., Seattle, WA) and SPSS v. 15.0 ${ }^{\mathrm{TM}}$ (SPSS Inc., Chicago, IL) software packages. The significance of all of the data was proven using the t-test, and $\mathrm{P}<0.05$ was considered significant.
Table I. Primary and secondary antibodies used for Western blotting.

\begin{tabular}{llll}
\hline $\begin{array}{l}\text { Antigen } \\
\text { specificity }\end{array}$ & \multicolumn{1}{c}{ Type } & Dilution & Vendor \\
\hline$\beta$-actin & Mouse monoclonal & $1: 2000$ & Sigma \\
Bcl-2 & Rabbit polyclonal & $1: 300$ & Abcam \\
CHOP & Rabbit polyclonal & $1: 400$ & Abcam \\
Bax & Rabbit polyclonal & $1: 300$ & Abcam \\
Caspase 12 & Rabbit polyclonal & $1: 500$ & Abcam \\
& & & \\
Rabbit IgG & Mouse monoclonal, & $1: 1000$ & Sigma \\
& peroxidase conjugated & & \\
& & & Sigma \\
Mouse IgG & Rabbit polyclonal, & $1: 1000$ & \\
& peroxidase conjugated & & \\
\hline
\end{tabular}

\section{Results}

siRNA-mediated silencing of mucin production. Capan-1 pancreatic carcinoma cells with a high mucin production were transfected with siRNA against various mucin genes. Transfection efficacy was determined by quantitative realtime RT-PCR after 24-120 h. All used siRNAs significantly reduced the expression of the respective mucin mRNA in a time-dependent manner, reaching a maximum suppression after $96 \mathrm{~h}$ (Fig. 1A). The knockdown of mucin genes was followed by a delayed reduction of the mucin concentration in cell culture supernatant (Fig. 1B). Here, silencing of MUC4 showed the most distinctive reduction of mucin secretion. We therefore used Capan-1 cells transfected with siRNA against MUC4, named sCapan-1, for all further experiments.

There were no differences in viability or proliferation between native Capan-1 or sCapan-1 cells as determined by ${ }^{3} \mathrm{H}$-thymidine incorporation (data not shown). Phase contrast microscopy revealed a thinner mucin margin surrounding sCapan-1 cells and time for trypsinisation was reduced compared to native cells ( 2 vs. $4 \mathrm{~min}$ at $37^{\circ} \mathrm{C}, 0.01 \%$ trypsin/EDTA).

Effects of gemcitabine and bortezomib on sCapan-1. As high levels of mucins have been associated with resistance to the pyrimidine analogue 5-fluorouracil (23), we investigated the effect of gemcitabine, the current standard pyrimidine analogue for pancreatic cancer, on native and sCapan-1 cells. ${ }^{3} \mathrm{H}$-thymidine incorporation showed a complete suppression of cell growth in native Capan-1 cells at gemcitabine concentrations of $2 \mu \mathrm{M}$ or higher. Interestingly, sCapan- 1 cells showed an increased sensitivity and growth suppression was already observed at $0.1 \mu \mathrm{M}$ gemcitabine (Fig. 2A). In parallel, apoptosis induction as determined by flow cytometry of sub-G1-events after propidium iodide staining showed a higher sensitivity to $2 \mu \mathrm{M}$ gemcitabine in sCapan-1 and reduced rates of apoptosis in native cells (Fig. 2B).

In contrast, native Capan-1 cells showed an enhanced sensitivity towards treatment with the proteasome inhibitor BTZ. Growth rate, as determined by ${ }^{3} \mathrm{H}$-thymidine incorporation, 
A
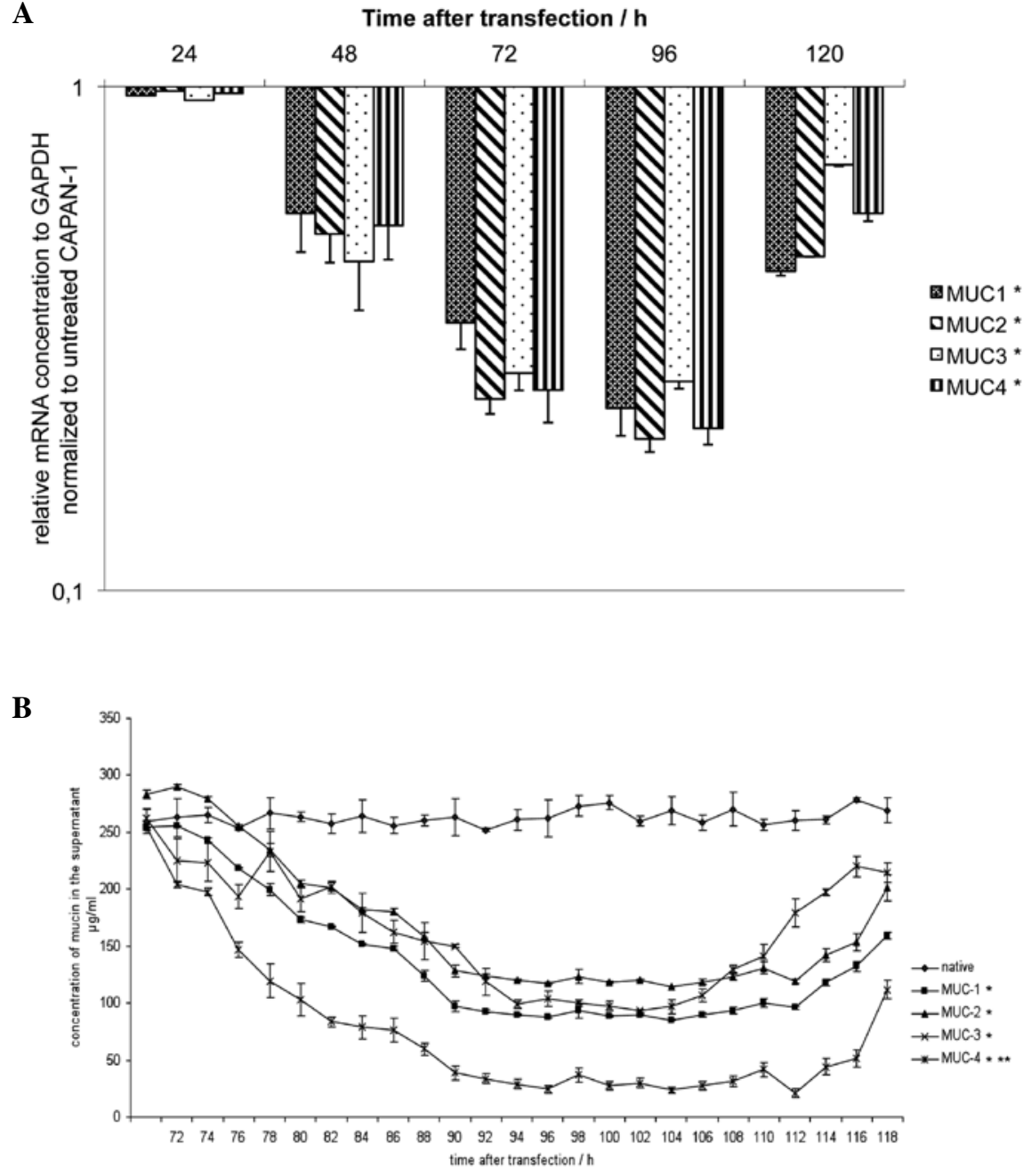

Figure 1. siRNA mediated silencing of mucin genes in Capan-1 cells. (A) Quantitative RT-PCR analysis of mRNA levels of mucin genes (MUC1 to MUC4) at 24-120 h after transfection showing a strong suppression especially at 72 and $96 \mathrm{~h}$. Results are normalized to GAPDH expression and expressed relative to untreated cells set at 1.0 . ${ }^{*} \mathrm{P}<0.01$ vs. native Capan-1. (B) Mucin concentration in cell culture supernatant is reduced after silencing of mucin genes compared to native Capan-1 cells. "P $<0.01$ vs. native Capan-1; ${ }^{* *} \mathrm{P}<0.01$ vs. MUC1, MUC2, MUC3.

was significantly suppressed (>90\%) in Capan-1 cells at $0.5 \mu \mathrm{M}$, while this level of growth reduction was achieved in sCapan-1 cells only at $2 \mu \mathrm{M}$ after $24 \mathrm{~h}$ (Fig. 2C). Paralleling these results, sCapan-1 cells also showed a delayed and diminished apoptosis response to BTZ compared to the parental cell line as shown by quantification of sub-diploid events after propidium iodide staining (Fig. 2D). Treatment with $1 \mu \mathrm{M} \mathrm{BTZ} \mathrm{lead} \mathrm{to} \mathrm{a}$ maximum of $\sim 40 \%$ apoptosis in native cells, while silenced cells reached only $25 \%$ after $36 \mathrm{~h}$ of incubation. Apoptotic cell death was confirmed by TUNEL staining (Fig. 2E). These findings indicate that mucin secretion influences differential pathways of chemotherapy response to either classical pyrimidine analogues or to novel targeted therapies like BTZ.

Bortezomib induces apoptosis via mitochondrial pathways. To address the underlying pathways of apoptosis induction, we examined the expression of the mitochondrial membrane proteins bax and bcl-2 in native and silenced Capan-1 cells after treatment with $1 \mu \mathrm{M}$ BTZ by quantitative RT-PCR . Pro-apoptotic bax was induced 6-fold in Capan-1, while the expression was reduced in sCapan-1 cells. Also anti-apoptotic bcl-2 was downregulated in both cell lines, a stronger suppres- sion was observed in native cells compared to sCapan-1, shifting the overall bax/bcl-2 ratio to 130.02 and 0.48 , respectively (Fig. 3A and B). These results were confirmed on the protein level by Western blot analysis (Fig. 3C). The functional relevance of the pro-apoptotic bax/bcl-2 ratio was further confirmed by showing an increased breakdown of the mitochondrial transmembrane potential $\Delta \Psi_{\mathrm{m}}$ by $\mathrm{DiOC}_{6}$ staining in native Capan-1 cells after BTZ treatment compared to sCapan-1 (Fig. 3D) which was significantly correlated to the apoptosis values determined by propidium iodide staining $\left(\mathrm{R}^{2}\right.$ $=0.87$ for Capan- 1 and $\mathrm{R}^{2}=0.74$ for sCapan-1). Furthermore, activity of the executioner caspases 3/7 and their activator caspase 8 was significantly higher in treated Capan-1 cells compared to sCapan-1 (Fig. 3D and E). Co-incubation with the pan-caspase inhibitor zVAD reduced this activation to control level in both cell lines.

Apoptotic response is independent of $N F \kappa B$ pathway inhibition. Inhibition of the transcription factor nuclear factor kappa $\mathrm{B}(\mathrm{NF \kappa} \mathrm{B})$ is considered as the main antitumor target of BTZ (9). We analyzed the activation of NFאB by an enzymatic mobility shift assay (EMSA, Fig. 4A). DNA binding of NFאB 


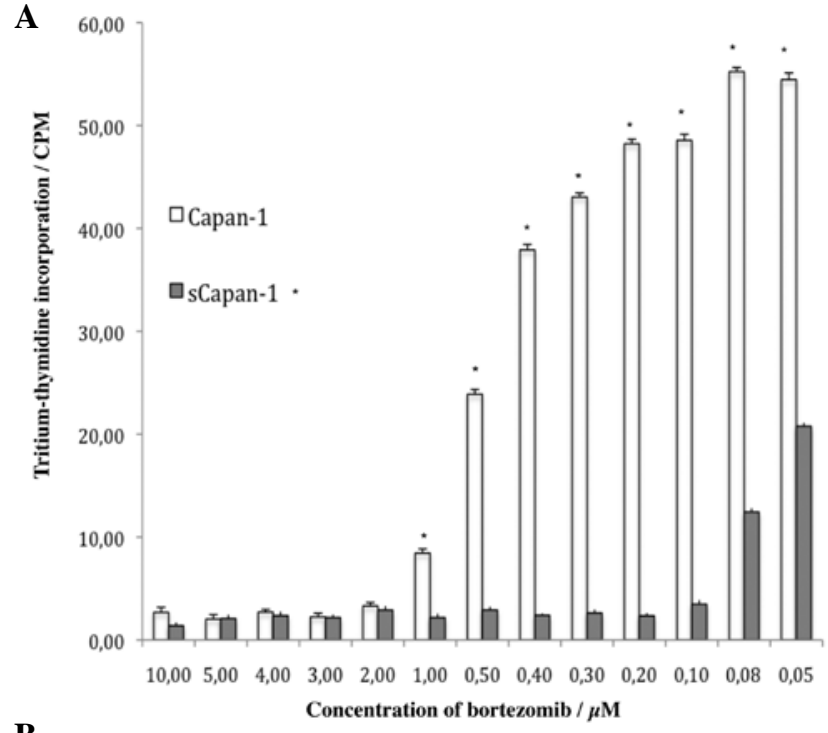

B

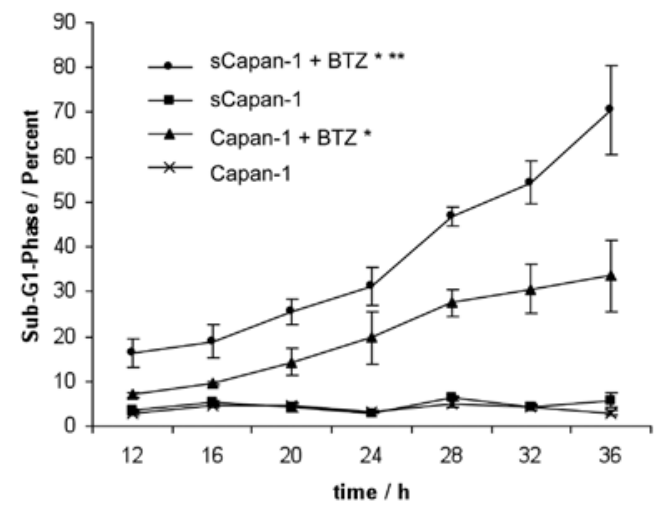

$\mathbf{E}$
C

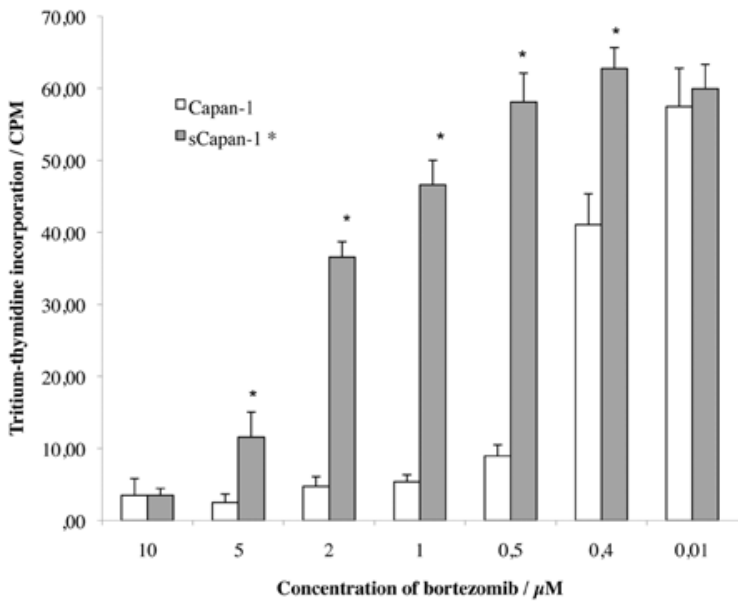

D

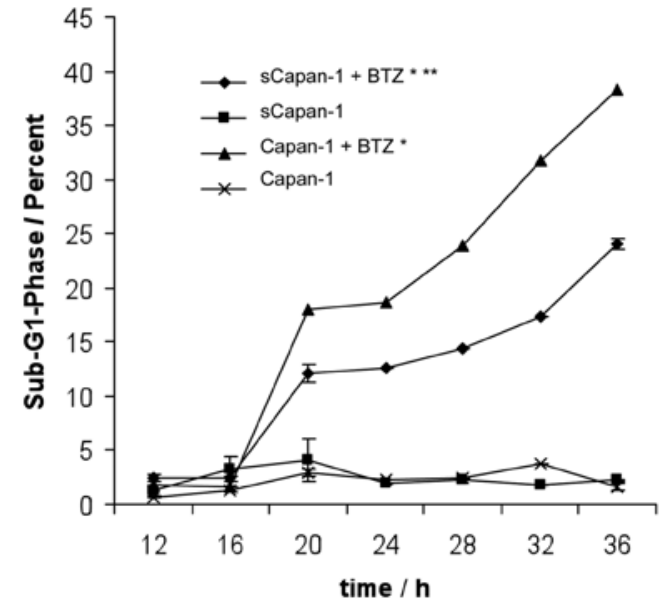

sCapan-1
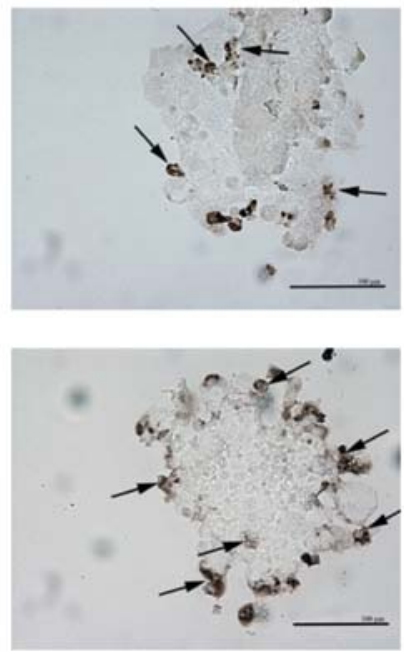

Figure 2. Differential effect of gemcitabine and bortezomib on cell viability and apoptosis in native and sCapan-1 cells. (A) Dose-dependent inhibition of cell proliferation in Capan-1 and sCapan-1 cells after 16-h treatment with gemcitabine. ${ }^{*} \mathrm{P}<0.01$ vs. sCapan-1. (B) Time-dependent induction of apoptosis by $2 \mu \mathrm{M}$ gemcitabine in Capan-1 and sCapan-1 cells. ${ }^{*} \mathrm{P}<0.01$ vs. untreated cells; ${ }^{* *} \mathrm{P}<0.01$ vs. Capan- $1+\mathrm{BTZ}$. (C) Dose-dependent inhibition of cell proliferation in Capan-1 and sCapan- 1 cells after 16-h treatment with BTZ. "P $<0.01$ vs. Capan-1. (D) Time-dependent induction of apoptosis by $2 \mu \mathrm{M}$ BTZ in Capan- 1 and sCapan-1 cells. ${ }^{*} \mathrm{P}<0.01$ vs. untreated cells; ${ }^{* *} \mathrm{P}<0.01$ vs. Capan-1 + BTZ E) TUNEL staining after 24-h treatment with $1 \mu \mathrm{M}$ BTZ. TUNEL positive cells are depicted by arrows.

was high in both untreated cell lines. NFkB activation was suppressed in both cell lines after treatment with $0.5 \mu \mathrm{M}$ BTZ for $20 \mathrm{~h}$. Yet, the DNA binding activity of NFkB is not neces- sarily paired with an increased transcription (32), which could also be influenced by other factors like RNAi related pathways. We therefore analyzed the mRNA level of I $\kappa$ B (nuclear 
A1

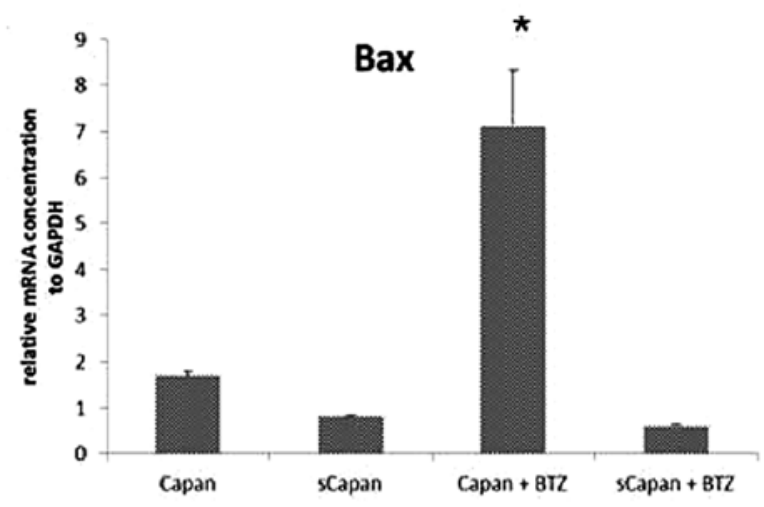

B

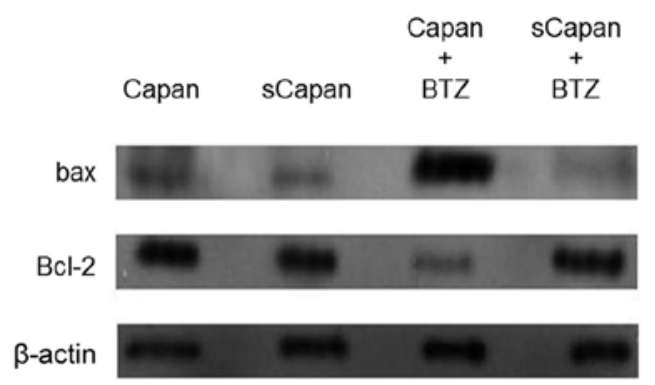

D

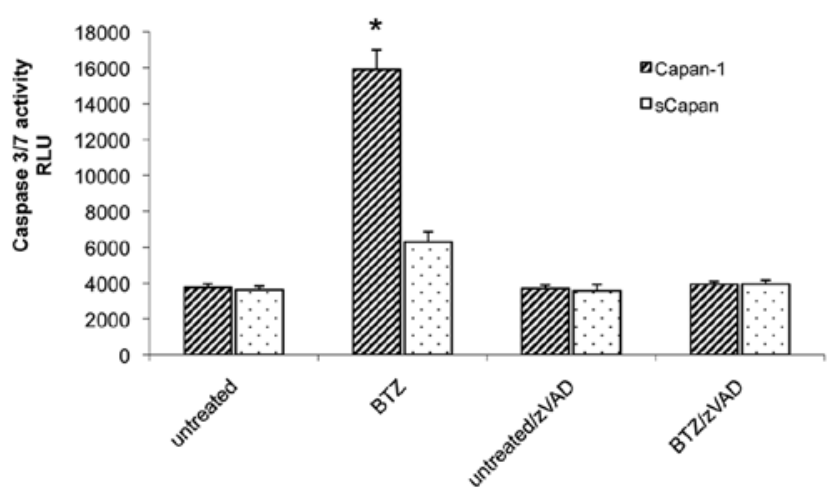

$\mathbf{E}$
A2
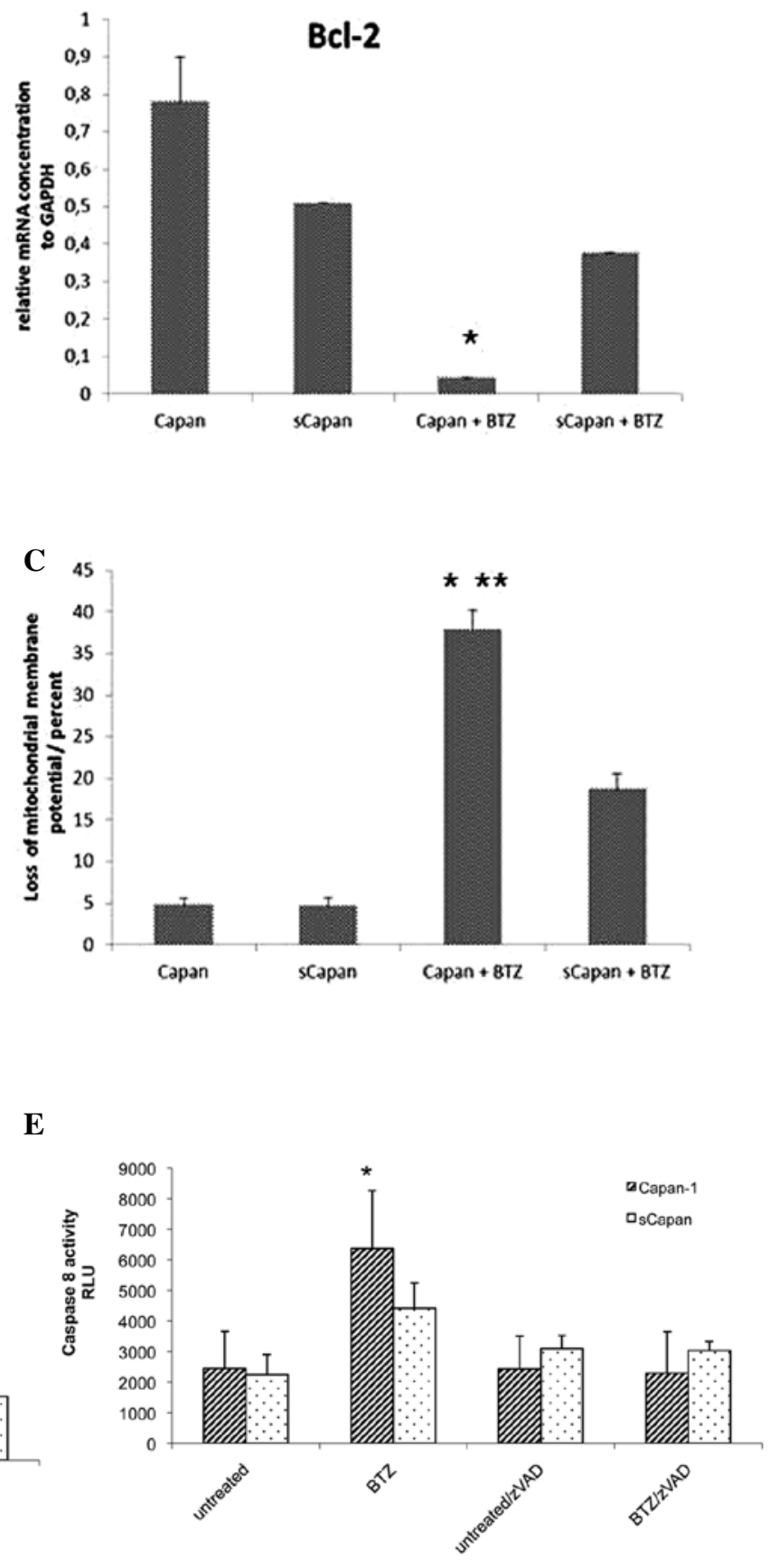

Figure 3. Analysis of apoptotic pathways in BTZ-treated Capan-1 and sCapan-1 cells. Quantitative RT-PCR shows (A) a strong upregulation of pro-apoptotic bax mRNA (1) in native Capan-1 cells after treatment with BTZ; "P<0.01 vs. untreated cells and sCapan + BTZ. Similarly, anti-apoptotic bcl-2 mRNA (2) is significantly reduced in these cells; ${ }^{*} \mathrm{P}<0.01$ vs. untreated cells and sCapan $+\mathrm{BTZ}$. (B) Representative Western blot analysis with $\beta$-actin as an internal standard confirmed the shift in the bax/bcl-2 ration towards apoptosis induction. (C) Flow cytometric analysis of mitochondrial transmembrane potential $\Delta \Psi_{\mathrm{m}}$ by $\mathrm{DiOC}_{6}$ staining after BTZ treatment shows a pronounced loss in intact mitochondria in native BTZ-treated Capan-1 cells; ${ }^{*} \mathrm{P}<0.01$ vs. untreated cells; ${ }^{* *} \mathrm{P}<0.01$ vs. sCapan-1 + BTZ. Under these conditions, the activity of the effector caspases $3 / 7$ (D) and of the initiator caspase 8 (E) are significantly ( $\left.{ }^{*}<0.01\right)$ increased as determined by a substrate cleavage assay. Cotreatment with the pan-caspase inhibitor zVAD reduced caspase activity to the level of untreated controls. Results are mean \pm SD of three independent experiments.

factor of kappa light polypeptide gene enhancer in B-cells inhibitor), an inhibitor of $\mathrm{NF} \kappa \mathrm{B}$ function (33). Although $\mathrm{NF} \kappa \mathrm{B}$ activation induces $\mathrm{I} \kappa \mathrm{B}$ transcription in an inhibitory feedback loop, we found a significant decrease in I $\mathrm{B}$ mRNA after BTZ treatment in both cell lines (Fig. 4B). The similar sensitivity towards NFкB inhibition in both cell lines is discrepant to the observed apoptosis induction in Capan-1 and sCapan-1 cells after BTZ treatment, indicating that additional pathways of cell death induction are involved.

Secretory activity sensitizes endoplasmic reticulum-mediated apoptosis by bortezomib. As protein and mucus secretion is linked to integrity of the endoplasmic reticulum (ER), we investigated if BTZ disturbs ER processes after silencing of 
A
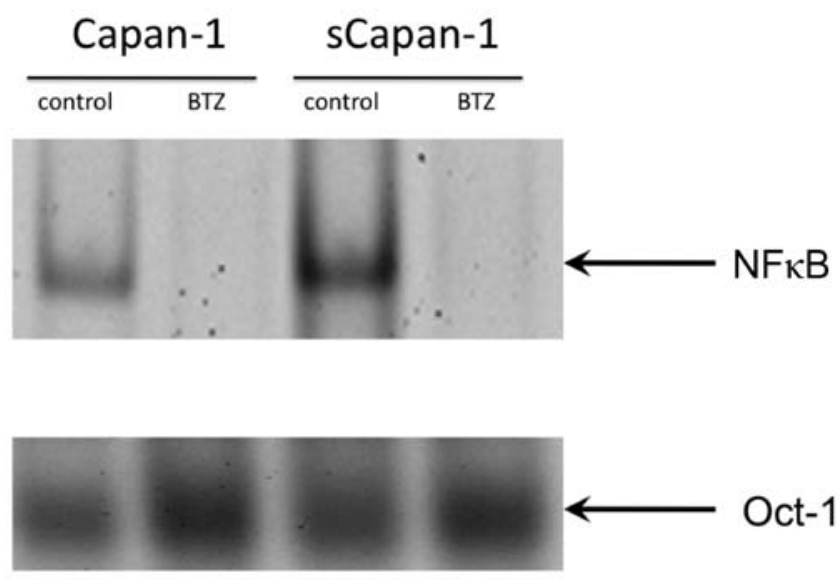

B

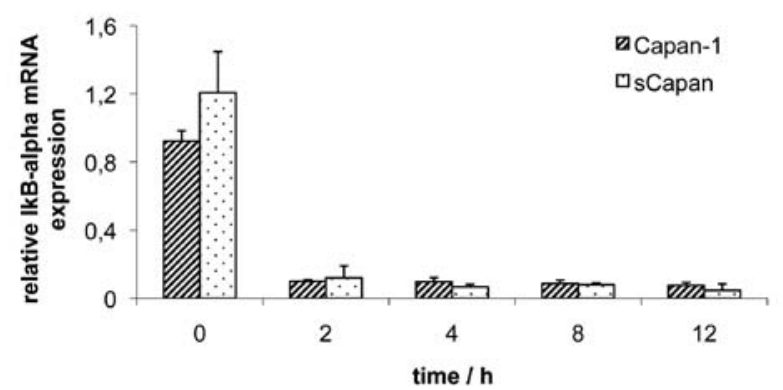

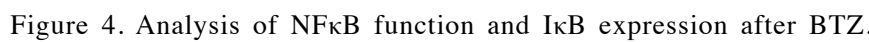
(A) Enzymatic mobility shift assay shows a significant reduction of NFKB DNA binding in both cell types after BTZ treatment, while the transcription factor Oct-1 was used as an internal control. (B) Quantitative RT-PCR of IкB mRNA shows a suppression in both cell lines after BTZ treatment. There were significant differences between Capan and sCapan.
MUC4 and activates the unfolded protein response (UPR) which could provide further insight into the observed differences in apoptosis induction between native and silenced Capan-1 cells.

ER stress and UPR are usually initiated by activation of the endonuclease activity of IRE1, which excises a 26-bp fragment from XBP-1 mRNA. In our hands, splicing of XBP-1 occurred in native Capan-1 cells but not in sCapan-1 cells after treatment with $1 \mu \mathrm{M}$ BTZ (Fig. 5A).

Western blot analysis showed a strong induction of $\mathrm{CHOP}$ expression after treatment with $1 \mu \mathrm{M}$ BTZ in Capan-1, but only a minor response in sCapan-1 cells. This was paralleled by a delayed induction of caspase 12 expression, the initiator caspase of the ER stress pathway, after $24 \mathrm{~h}$ in BTZ-sensitive Capan-1 cells (Fig. 5B). Interestingly, we could not detect significant changes in protein or mRNA levels of ER stress related chaperones like $\mathrm{BiP}$, which may be due to high levels of BiP already in untreated control cells (data not shown). These results highlight the capability of BTZ to induce additional apoptotic pathways in pancreatic cancer cells beyond the previously described NFKB signalling.

\section{Discussion}

Advanced pancreatic cancer is characterized by a high intrinsic resistance to conventional chemotherapies and novel effective and tolerable treatment regimens are therefore urgently needed (3). Recent data suggest that production of mucin molecules may be directly linked to the inhibition of apoptosis in pancreatic cancer cells (34). Mucins are a family of high molecular weight proteins and are often aberrantly expressed in cancers (35-37). In addition to altering surface properties of tumor cells, several mucin molecules also have intracellular domains that are directly connected to oncogenic signalling pathways $(38,39)$.

A

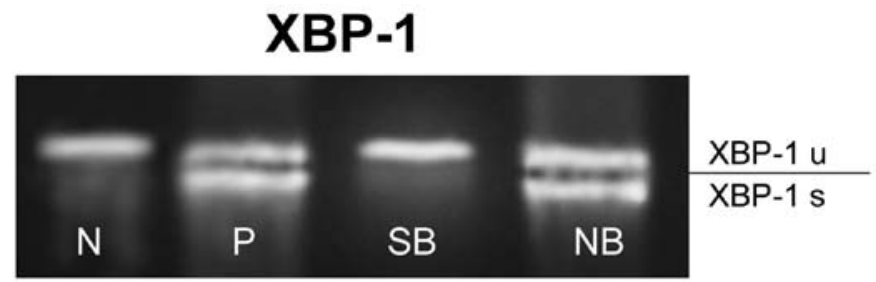

B

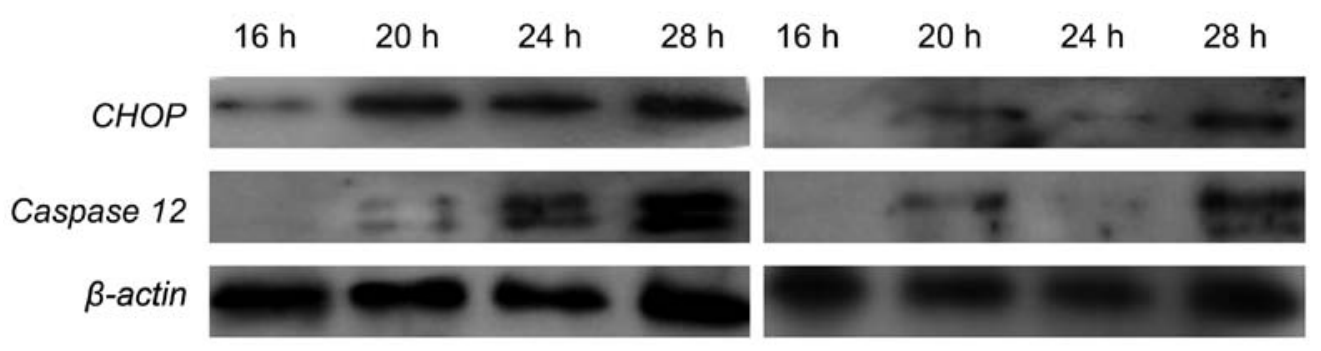

Figure 5. Silencing of mucin genes inhibits ER stress mediated caspase activation. (A) PCR analysis of Xbp-1 splicing after BTZ treatment in native and silenced Capan-1 cells. (B) Western blot analysis of CHOP and caspase 12 expression. 
MUC4 has been reported to be expressed in pancreatic adenocarcinomas but not in adjacent normal tissue $(40,41)$ and was proposed to be a novel tumor marker for this disease as the prognosis of patients with high MUC4 expression was significantly reduced (42). The intracellular domain of MUC4 contains an amino acid sequence that acts as a direct stimulating ligand for the tyrosine kinase receptor erbB2 $(43,44)$. ErbB2 itself has been shown to be significantly overexpressed in human pancreatic ductal adenocarcinomas $(45,46)$ and to regulate inflammatory and cell viability pathways, e.g., by NFאB (47). MUC4 has thus been demonstrated to stimulate proliferation pathways and to inhibit apoptosis induced by chemotherapeutic drugs in different cancer cell lines $(27,34,48)$.

We have previously established RNA interference based strategies to inhibit oncogene function in pancreatic cancer models $(28,49)$ and others have shown that the knockdown of MUC4 interferes with the above describe tumorigenic pathways $(24,26,27)$. We have shown here that the siRNAmediated knockdown of different mucin genes leads to a significant and sustained suppression of both mucin mRNA and protein and interferes with sensitivity to gemcitabine and BTZ. In our hands, silencing of MUC4 increases the sensitivity towards treatment with the pyrimidine analogue gemcitabine, confirming the observation that the inhibition of mucin production by benzyl- $\alpha$-GalNAc restores sensitivity of Capan-1 cells to 5-fluorouracil (23).

The proteasome inhibitor BTZ has been shown to possess a strong anti-tumor activity against pancreatic cancer cells in vitro $(8,50)$. Surprisingly, BTZ did not prove to be effective in in vivo models or early clinical trials of pancreatic cancer $(7,8,51)$. In our study, BTZ inhibited proliferation and induced apoptosis in both native and silenced Capan-1 cells with a significantly stronger effect in MUC4-expressing native Capan-1 cells. These results are in line with our previous findings that a high immunoglobulin production sensitizes myeloma cells to BTZ treatment (22). In native Capan-1 cells, BTZ induces mitochondrial apoptosis as shown by a shift of the bax/bcl-2 ratio and breakdown of $\Delta \Psi_{\mathrm{m}}$, but is also capable of activating novel alternative pathways of cell death. As described in the myeloma model (22), we also found a strong activation of the unfolded protein response (UPR) and endoplasmic reticulum stress (ER) stress pathways especially in native Capan-1 cells as demonstrated by splicing of Xbp-1 and increased expression of CHOP. The misfolding of proteins activates the UPR by enhancing chaperone activity and by ubiquitinating misfolded proteins for proteasomal degradation. These pathways have been described for BTZ treated pancreatic cancer cells $(19,20)$ and our results support the hypothesis that the secretory activity, i.e., the degree of mucin production, regulates the apoptotic response by ER stress. This hypothesis is further supported by the notion that pancreatic epithelial cells have a high protein synthesis capacity and therefore possess an extensive ER which sensitizes these cells to UPR (52-54).

Although inhibition of $\mathrm{NF \kappa B}$ signalling is currently conceived as the main effect of BTZ on apoptosis and cell growth (9) we did not detect significant differences between the two investigated cell lines in either $\mathrm{NF} \kappa \mathrm{B}$ or I $\kappa \mathrm{B}$ signalling that could explain the observed differences in BTZ-induced apoptosis. It was previously described that BTZ does not influ- ence the transcription rate of $\mathrm{NF} \kappa \mathrm{B}$ family members or $\mathrm{p} 53$ but does instead induce genes related to UPR or to cellular protein metabolism, e.g., chaperones of the heat shock protein family (55). This finding further highlights the importance of UPR and the protein synthesis activity in our experimental setting.

In conclusion, our results show that the secretory activity of pancreatic cancer cells determines the sensitivity towards either conventional chemotherapeutic agents like pyrimidine analogues or to novel agents like BTZ. The stratification of patients according to the expression of MUC genes, which have already been shown to be a good diagnostic and prognostic biomarker, could therefore contribute to an improved chemotherapy response in these patients and also serve as a therapeutic biomarker. Strategies to inhibit MUC genes, e.g., by RNAi based approaches or by application of benzyl- $\alpha-$ GalNAc could therefore provide new therapy options for this disease.

\section{Acknowledgements}

This study was supported by a grant of the interdisciplinary center for clinical research (IZKF) at the University Hospital Erlangen to T.T.W. and the Löwel-Foundation, Bamberg, Germany. M.O. was supported by a Research Grant of the University Medical Center Giessen and Marburg (UKGM). We thank Astrid Taut and Isabel Zeitträger for their excellent technical assistance.

\section{References}

1. Jemal A, Siegel R, Ward E, et al: Cancer statistics, 2008. CA Cancer J Clin 58: 71-96, 2008.

2. Carpelan-Holmstrom M, Nordling S, Pukkala E, et al: Does anyone survive pancreatic ductal adenocarcinoma? A nationwide study re-evaluating the data of the Finnish Cancer Registry. Gut 54: 385-387, 2005 .

3. Ghaneh P, Costello E and Neoptolemos JP: Biology and management of pancreatic cancer. Postgrad Med J 84: 478-497, 2008.

4. Sultana A, Tudur Smith C, Cunningham D, Starling N, Neoptolemos JP and Ghaneh P: Meta-analyses of chemotherapy for locally advanced and metastatic pancreatic cancer: results of secondary end points analyses. Br J Cancer 99: 6-13, 2008.

5. Li D, Xie K, Wolff R and Abbruzzese JL: Pancreatic cancer. Lancet 363: 1049-1057, 2004.

6. Sterz J, von Metzler I, Hahne JC, et al: The potential of proteasome inhibitors in cancer therapy. Expert Opin Investig Drugs 17: 879-895, 2008.

7. Alberts SR, Foster NR, Morton RF, et al: PS-341 and gemcitabine in patients with metastatic pancreatic adenocarcinoma: a North Central Cancer Treatment Group (NCCTG) randomized phase II study. Ann Oncol 16: 1654-1661, 2005.

8. Marten A, Zeiss N, Serba S, Mehrle S, von Lilienfeld-Toal M and Schmidt J: Bortezomib is ineffective in an orthotopic mouse model of pancreatic adenocarcinoma. Mol Cancer Ther 7: 3624-3631, 2008.

9. Sebens S, Arlt A and Schafer H: NF-kappaB as a molecular target in the therapy of pancreatic carcinoma. Recent Results Cancer Res 177: 151-164, 2008 .

10. Hamacher R, Schmid RM, Saur D and Schneider G: Apoptotic pathways in pancreatic ductal adenocarcinoma. Mol Cancer 7: $64,2008$.

11. Holcomb B, Yip-Schneider M and Schmidt CM: The role of nuclear factor kappaB in pancreatic cancer and the clinical applications of targeted therapy. Pancreas 36: 225-235, 2008.

12. Scheidereit C: IkappaB kinase complexes: gateways to NF-kappaB activation and transcription. Oncogene 25: 6685-6705, 2006.

13. Perkins ND: Integrating cell-signalling pathways with NF-kappaB and IKK function. Nat Rev Mol Cell Biol 8: 49-62, 2007.

14. Wang W, Abbruzzese JL, Evans DB, Larry L, Cleary KR and Chiao PJ: The nuclear factor-kappa B RelA transcription factor is constitutively activated in human pancreatic adenocarcinoma cells. Clin Cancer Res 5: 119-127, 1999. 
15. Weichert W, Boehm M, Gekeler V, et al: High expression of $\mathrm{RelA} / \mathrm{p} 65$ is associated with activation of nuclear factor-kappaBdependent signaling in pancreatic cancer and marks a patient population with poor prognosis. Br J Cancer 97: 523-530, 2007.

16. Arlt A, Vorndamm J, Breitenbroich M, et al: Inhibition of NF-kappaB sensitizes human pancreatic carcinoma cells to apoptosis induced by etoposide (VP16) or doxorubicin. Oncogene 20: 859-868, 2001

17. Muerkoster S, Arlt A, Witt M, et al: Usage of the NF-kappaB inhibitor sulfasalazine as sensitizing agent in combined chemotherapy of pancreatic cancer. Int J Cancer 104: 469-476, 2003.

18. Thomas RP, Farrow BJ, Kim S, May MJ, Hellmich MR and Evers BM: Selective targeting of the nuclear factor-kappaB pathway enhances tumor necrosis factor-related apoptosisinducing ligand-mediated pancreatic cancer cell death. Surgery 132: 127-134, 2002

19. Nawrocki ST, Carew JS, Dunner K Jr, et al: Bortezomib inhibits PKR-like endoplasmic reticulum (ER) kinase and induces apoptosis via ER stress in human pancreatic cancer cells. Cancer Res 65: 11510-11519, 2005.

20. Nawrocki ST, Carew JS, Pino MS, et al: Bortezomib sensitizes pancreatic cancer cells to endoplasmic reticulum stress-mediated apoptosis. Cancer Res 65: 11658-11666, 2005.

21. Forstner G: Signal transduction, packaging and secretion of mucins. Annu Rev Physiol 57: 585-605, 1995.

22. Meister S, Schubert U, Neubert K, et al: Extensive immunoglobulin production sensitizes myeloma cells for proteasome inhibition. Cancer Res 67: 1783-1792, 2007.

23. Kalra AV and Campbell RB: Mucin overexpression limits the effectiveness of 5-FU by reducing intracellular drug uptake and antineoplastic drug effects in pancreatic tumours. Eur J Cancer 45: 164-173, 2009

24. Chaturvedi P, Singh AP, Chakraborty S, et al: MUC4 mucin interacts with and stabilizes the HER2 oncoprotein in human pancreatic cancer cells. Cancer Res 68: 2065-2070, 2008.

25. Tinder TL, Subramani DB, Basu GD, et al: MUC1 enhances tumor progression and contributes toward immunosuppression in a mouse model of spontaneous pancreatic adenocarcinoma. J Immunol 181: 3116-3125, 2008.

26. Singh AP, Moniaux N, Chauhan SC, Meza JL and Batra SK Inhibition of MUC4 expression suppresses pancreatic tumor cell growth and metastasis. Cancer Res 64: 622-630, 2004.

27. Chaturvedi P, Singh AP, Moniaux N, et al: MUC4 mucin potentiates pancreatic tumor cell proliferation, survival, and invasive properties and interferes with its interaction to extracellular matrix proteins. Mol Cancer Res 5: 309-320, 2007.

28. Ocker M, Neureiter D, Lueders M, et al: Variants of bcl-2 specific siRNA for silencing antiapoptotic bcl-2 in pancreatic cancer. Gut 54: 1298-1308, 2005.

29. Zopf S, Neureiter D, Bouralexis $\mathrm{S}$, et al: Differential response of p53 and p21 on HDAC inhibitor-mediated apoptosis in HCT116 colon cancer cells in vitro and in vivo. Int J Oncol 31: 1391-1402, 2007.

30. Di Fazio P, Schneider-Stock R, Neureiter D, et al: The pan-deacetylase inhibitor panobinostat inhibits growth of hepatocellular carcinoma models by alternative pathways of apoptosis. Cell Oncol 32: 285-300, 2010.

31. Voll RE, Jimi E, Phillips RJ, et al: NF-kappa B activation by the pre-T cell receptor serves as a selective survival signal in $\mathrm{T}$ lymphocyte development. Immunity 13: 677-689, 2000

32. Hoffmann A, Natoli G and Ghosh G: Transcriptional regulation via the NF-kappaB signaling module. Oncogene 25: 6706-6716, 2006.

33. Solt LA and May MJ: The IkappaB kinase complex: master regulator of NF-kappaB signaling. Immunol Res 42: 3-18, 2008

34. Workman HC, Sweeney C and Carraway KL III: The membrane mucin Muc4 inhibits apoptosis induced by multiple insults via ErbB2-dependent and ErbB2-independent mechanisms. Cancer Res 69: 2845-2852, 2009.

35. Hattrup CL and Gendler SJ: Structure and function of the cell surface (tethered) mucins. Annu Rev Physiol 70: 431-457, 2008
36. Terada T, Ohta T, Sasaki M,Nakanuma Y and Kim YS: Expression of MUC apomucins in normal pancreas and pancreatic tumours. J Pathol 180: 160-165, 1996.

37. Yonezawa $\mathrm{S}$ and Sato E: Expression of mucin antigens in human cancers and its relationship with malignancy potential. Pathol Int 47: 813-830, 1997.

38. Carraway KL III, Funes M, Workman HC and Sweeney C: Contribution of membrane mucins to tumor progression through modulation of cellular growth signaling pathways. Curr Top Dev Biol 78: 1-22, 2007

39. Singh PK and Hollingsworth MA: Cell surface-associated mucins in signal transduction. Trends Cell Biol 16: 467-476, 2006.

40. Andrianifahanana M, Moniaux N, Schmied BM, et al: Mucin (MUC) gene expression in human pancreatic adenocarcinoma and chronic pancreatitis: a potential role of MUC4 as a tumor marker of diagnostic significance. Clin Cancer Res 7: 40334040, 2001.

41. Swartz MJ, Batra SK, Varshney GC, et al: MUC4 expression increases progressively in pancreatic intraepithelial neoplasia. Am J Clin Pathol 117: 791-796, 2002.

42. Yonezawa S, Goto M, Yamada N, Higashi $M$ and Nomoto $M$ Expression profiles of MUC1, MUC2, and MUC4 mucins in human neoplasms and their relationship with biological behavior. Proteomics 8: 3329-3341, 2008.

43. Jepson S, Komatsu M, Haq B, et al: Muc4/sialomucin complex, the intramembrane ErbB2 ligand, induces specific phosphorylation of ErbB2 and enhances expression of p27(kip), but does not activate mitogen-activated kinase or protein kinaseB/Akt pathways. Oncogene 21: 7524-7532, 2002.

44. Carraway KL III, Rossi EA, Komatsu M, et al: An intramembrane modulator of the ErbB2 receptor tyrosine kinase that potentiates neuregulin signaling. J Biol Chem 274: 5263-5266, 1999.

45. Pryczynicz A, Guzinska-Ustymowicz K, Kemona A and Czyzewska J: Expression of EGF and EGFR strongly correlates with metastasis of pancreatic ductal carcinoma. Anticancer Res 28: 1399-1404, 2008.

46. Safran H, Steinhoff M, Mangray S, et al: Overexpression of the HER-2/neu oncogene in pancreatic adenocarcinoma. Am J Clin Oncol 24: 496-499, 2001

47. Algul $\mathrm{H}$, Wagner M, Lesina $\mathrm{M}$ and Schmid RM: Overexpression of ErbB2 in the exocrine pancreas induces an inflammatory response but not increased proliferation. Int J Cancer 121: 1410$1416,2007$.

48. Miyahara N, Shoda J, Ishige K, et al: MUC4 interacts with ErbB2 in human gallbladder carcinoma: potential pathobiological implications. Eur J Cancer 44: 1048-1056, 2008.

49. Okamoto K, Ocker M, Neureiter D, et al: bcl-2-specific siRNAs restore gemcitabine sensitivity in human pancreatic cancer cells. J Cell Mol Med 11: 349-361, 2007.

50. Shah SA, Potter MW, McDade TP, et al: $26 \mathrm{~S}$ proteasome inhibition induces apoptosis and limits growth of human pancreatic cancer. J Cell Biochem 82: 110-122, 2001.

51. Sloss CM, Wang F, Liu R, et al: Proteasome inhibition activates epidermal growth factor receptor (EGFR) and EGFR-independent mitogenic kinase signaling pathways in pancreatic cancer cells. Clin Cancer Res 14: 5116-5123, 2008.

52. Oyadomari S, Araki E and Mori M: Endoplasmic reticulum stress-mediated apoptosis in pancreatic beta-cells. Apoptosis 7: 335-345, 2002.

53. Harding HP, Zeng H, Zhang Y, et al: Diabetes mellitus and exocrine pancreatic dysfunction in perk $^{-/}$mice reveals a role for translational control in secretory cell survival. Mol Cell 7: 1153-1163, 2001.

54. Zhang P, McGrath B, Li S, et al: The PERK eukaryotic initiation factor 2 alpha kinase is required for the development of the skeletal system, postnatal growth, and the function and viability of the pancreas. Mol Cell Biol 22: 3864-3874, 2002.

55. Tang ZY, Wu YL, Gao SL and Shen HW: Effects of the proteasome inhibitor bortezomib on gene expression profiles of pancreatic cancer cells. J Surg Res 145: 111-123, 2008. 